\title{
Superluminescent diode with a broadband gain based on self- assembled InAs quantum dots and segmented contacts for an optical coherence tomography light source
}

Nobuhiko Ozaki, ${ }^{1,2, a), b)}$ David T. D. Childs, ${ }^{1}$ Jayanta Sarma, ${ }^{1}$ Timothy S. Roberts, ${ }^{1}$ Takuma Yasuda, ${ }^{2}$ Hiroshi Shibata, ${ }^{2}$ Hirotaka Ohsato, ${ }^{3}$ Eiichiro Watanabe, ${ }^{3}$

Naoki Ikeda, ${ }^{3}$ Yoshimasa Sugimoto, ${ }^{3}$ and Richard A. Hogg ${ }^{1}$

${ }^{1}$ Department of Electronic and Electrical Engineering, University of Sheffield, Sheffield S3 7HQ, UK

${ }^{2}$ Faculty of Systems Engineering, Wakayama University, Wakayama 640-8510, Japan

${ }^{3}$ National Institute for Materials Science (NIMS), Tsukuba, Ibaraki 305-0047, Japan

\section{Abstract}

We report a broadband-gain superluminescent diode (SLD) based on self-assembled InAs quantum dots (QDs) for application in a high-resolution optical coherence tomography (OCT) light source. Four InAs QD layers, with sequentially shifted emission wavelengths achieved by varying the thickness of the $\mathrm{In}_{0.2} \mathrm{Ga}_{0.8} \mathrm{As}$ strain-reducing capping layers, were embedded in a conventional p-n heterojunction comprising GaAs and AlGaAs layers. A ridge-type waveguide with segmented contacts was formed on the grown wafer, and an ascleaved 4-mm-long chip (QD-SLD) was prepared. The segmented contacts were effective in applying a high injection current density to the QDs and obtaining emission from excited states of the QDs, resulting in an extension of the bandwidth of the electroluminescence spectrum. In addition, gain spectra deduced with the segmented contacts indicated a broadband smooth positive gain region spanning $160 \mathrm{~nm}$. Furthermore, OCT imaging with the fabricated QD-SLD was performed, and OCT images with an axial resolution of $\sim 4 \mu \mathrm{m}$ in air were obtained. These results demonstrate the effectiveness of the QD-SLD with segmented contacts as a high-resolution OCT light source.

\footnotetext{
a) Electronic mail: ozaki@sys.wakayama-u.ac.jp

b) This research was partly performed while N. Ozaki was at The University of Sheffield, Sheffield, S3 7HQ, UK.
} 


\section{INTRODUCTION}

Near-infrared (NIR) broadband (low-coherence) light has been utilized as a useful probe for non-invasive cross-sectional biological and medical imaging system: optical coherence tomography $(\mathrm{OCT})^{1}$. OCT relies upon low-coherence interferometry, and the axial resolution of OCT is governed by the coherence length of the light source ${ }^{2}$, which is given by the formula $0.44 \times \lambda_{0}{ }^{2} / \Delta \lambda$ for a low-coherence light with a Gaussian spectral shape. Where the peak in emission is centered at $\lambda_{0}$ with a bandwidth of $\Delta \lambda$. As the application is in medical imaging, the center wavelength, $\lambda_{0}$, is preferably in the NIR wavelength range as NIR light offers deep penetration into biological samples, and a large imaging depth can be obtained ${ }^{3}$. Thus, a large bandwidth NIR light source is required to obtain an OCT image with high axial resolution and large penetration depth. As a specific criterion, a resolution of 3-5 $\mu \mathrm{m}$ is necessary to distinguish single cells ${ }^{4}$. In commercial OCT systems, a semiconductor-based superluminescent diode (SLD) is often used as a broadband light source. A SLD exhibits amplified spontaneous emission providing a broader spectral emission range than a laser diode and higher emission power and brightness than a light-emitting diode. However, it is difficult to broaden the emission bandwidth of a NIR SLD beyond 100 nanometers, which is one of the reasons for the limitation of the axial resolution in an OCT image to around $\sim 10$ $\mu \mathrm{m}$. Although alternative light sources have been developed for realizing high-resolution OCT, such as the use of a short-pulsed femtosecond laser ${ }^{5}$ or supercontinuum generation in a photonic crystal fiber ${ }^{6}$, a SLD still offers the advantages of being a compact, robust, and inexpensive device compared with alternative light sources.

To overcome the difficulty of obtaining a broadband-gain SLD, self-assembled quantum dots $(\mathrm{QDs})^{7}$ have been recently recognized as an ideal light-emitting material instead of a conventional quantum well (QW) or bulk semiconductor. A QD is a three-dimensionally quantum confined structure and has a unique configuration of electron states and discrete 
delta-function-like density of states (DOS) ${ }^{8}$. The states can be easily filled with supplied carriers, and the emission bandwidth of the QD should be extended in accordance with their discrete bandgap energies: the ground state (GS), $1^{\text {st }}$ excited state (ES1), and $2^{\text {nd }}$ excited state (ES2). In addition, self-assembled InAs QDs on a GaAs substrate, which are grown via the strain induced by a lattice mismatch between InAs and GaAs (Stranski-Krastanov growth mode $)^{9,10}$, are particularly suitable to emit a broadband spectrum in the NIR region because of their size and compositional distributions in the ensemble. A SLD based on InAs QDs (QDSLD) was first proposed by Wang et al. ${ }^{11}$ and has been intensively studied ${ }^{12-21}$. We have fabricated a QD-SLD based on InAs QDs with controlled emission wavelengths ${ }^{21}$ and demonstrated OCT imaging using the developed QD-SLD ${ }^{22}$. However, the bandwidth of the electroluminescence (EL) was limited because the emission was limited to that from GS and ES1 due to a limited injection current density $(J)$ through the use of a single contact electrode.

In this work, we thus developed a QD-based SLD, which includes multiple QD layers with deliberately shifted emission wavelengths, with segmented contacts to allow an increase in $J$ locally within the device and to exploit emissions from higher energy states of the QDs, i.e., ES2. The objective of this work was to realize a further broadened and dipless EL spectrum suitable for a high-resolution OCT light source. In addition, we measured gain spectra from the fabricated SLD using the segmented contact method ${ }^{23}$ to examine the gain bandwidth. Furthermore, OCT imaging with the fabricated QD-SLD was performed to evaluate its potential use in an OCT light source.

\section{EXPERIMENTAL DETAILS}

\section{A. Sample preparation}


Four stacked InAs QD layers capped with $\operatorname{In}_{0.2} \mathrm{Ga}_{0.8} \mathrm{As}$ layers of various thickness were grown by molecular beam epitaxy on an n+-GaAs (001) substrate, as illustrated in Fig. 1(a). All the QD layers were grown with a supply of InAs of 2.0 monolayers, where the density of the grown QDs was $2-4 \times 10^{10} \mathrm{~cm}^{-2}$. The $\mathrm{In}_{0.2} \mathrm{Ga}_{0.8}$ As layer deposited on each QD layer was used as a strain-reducing layer (SRL) ${ }^{24}$ to control the emission center wavelength of the QDs by varying its thickness ${ }^{25}: 0,1,2$, and $4 \mathrm{~nm}$. A 240-nm-thick GaAs waveguide layer including the QD layers was optically and electronically confined within $1.5-\mu \mathrm{m}$-thick p-/n$\mathrm{Al}_{0.35} \mathrm{Ga}_{0.65} \mathrm{As}$ cladding layers. The p-/n-cladding layers were doped at approximately $2.0 \times$ $10^{17} \mathrm{~cm}^{-3}$ by Be and Si, respectively. A 300-nm-thick $\mathrm{p}^{+}$-GaAs (doped: $\sim 1 \times 10^{19} \mathrm{~cm}^{-3}$ ) layer was grown on the upper p-cladding layer as a contact layer to the electrode.

A ridge waveguide (RWG) was fabricated normal to cleaved facets with a height of 1.4 $\mu \mathrm{m}$ and width of $5 \mu \mathrm{m}$ on the $\mathrm{p}$-side surface of the grown wafer using photolithography and inductively coupled plasma reactive ion etching techniques. An insulation film of 200-nmthick $\mathrm{SiO}_{2}$ was deposited on the surface using plasma-enhanced chemical vapor deposition, followed by wet-etching of selective areas (segments) on the RWG with buffered hydrofluoric acid solution. Subsequently, electrodes were formed by depositing Ti/Au (pcontact) and $\mathrm{Ni} / \mathrm{AuGe} / \mathrm{Ni} / \mathrm{Au}$ (n-contact), and rapid thermal annealing was performed. To form segmented contacts, the electrode of the p-contact was divided into four 1-mm-long electrically isolated segments via the lift-off process, as shown in Fig. 1(b). The contact gap lengths between the segments were approximately $40 \mu \mathrm{m}$. Both edges of the RWG were cleaved to form a 4-mm-long chip, and no anti-reflective (AR) coating was applied to the cleaved edges.

\section{B. EL and gain spectra measurement}


EL emission spectra from an edge of the fabricated RWG were measured at room temperature. Various injection currents (Is) were applied to the contact nearest to the edge (segment 1). The emission light was collected with an optical lensed fiber and was detected with an optical spectrum analyzer, which performs spectrum measurements through tunable bandpass filtering with a monochromator. The coupling loss into the optical lensed fiber was approximately $10 \mathrm{~dB}$.

The gain spectrum was obtained using the segmented contact method. Based on the following formula ${ }^{23}$,

$$
G=\frac{1}{L} \ln \left(\frac{P(J, 2 L)}{P(J, L)}-1\right),
$$

the gain and absorption spectra as a function of wavelength were determined. $P(J, L)$ and $P(J$, $2 L$ ) indicate the EL intensities obtained from the RWG lengths of $L$ and $2 L$, respectively, under the same $J$. Figures 2(a) and 2(b) present schematic diagrams of the device drive geometries; the current was injected into segment 1 only to measure $P(J, L)$, and both segments 1 and 2 were injected with identical $J$ to measure $P(J, 2 L)$.

The un-pumped segments are expected to act as absorber elements during the EL measurements and prevent lasing in the device. This feature of the segmented contact can be effective in obtaining a broadband SLD emission even though the RWG has a simple straight structure and no AR coating is formed on the facets.

\section{Spectral-domain OCT imaging}

A spectral-domain (SD)-OCT ${ }^{26}$ system was utilized to evaluate the fabricated chip as an OCT light source. The SD-OCT, which is categorized as a Fourier-domain OCT $^{27,28}$, enables a spatial reflectivity distribution to be obtained against the depth of a sample along with the optical axis through an inverse Fourier-transformed (IFT) spectrum of interference between 
reflections from the sample and a reference mirror. As schematically illustrated in Fig. 3, the OCT setup consisted of the QD-SLD as a light source, a reference arm, a sample arm, and a spectrometer. Light from the QD-SLD was split by a 50:50 coupler and introduced into the reference and sample arms. Then, the reflected light from the two arms was recombined by the 50:50 coupler, and the interference signal was measured by the spectrometer. The interference signal sampled as a function of the wavelength was rescaled to the wavenumber, $I(k)$, by the linear interpolation method. The zero-filling method was also used to increase the number of data points on both sides of the spectrum, $I(k)^{29}$. Subsequently, the IFT spectrum and depth profile of the reflectivity in the sample along with the optical axis was obtained. By collecting the depth profile with a lateral scanning of the sample, a 2D OCT image was obtained.

\section{Results and discussion}

\section{A. EL measurements}

Figure 4 presents the EL spectra obtained from the fabricated chip under various $I \mathrm{~s}=1-$ $150 \mathrm{~mA}$, corresponding to current densities of $J=20-3000 \mathrm{~A} / \mathrm{cm}^{2}$, which were applied to segment 1. As clearly observed in the series of spectra with injected currents (Figs. 3(a)-(c)), the spectral shape and center wavelength varied with the increased current. Initially, 1230nm-centered emission spanning approximately $1100-1300 \mathrm{~nm}$ (the bandwidth was below 100 $\mathrm{nm})$ was exhibited at lower Is ( 3 mA), as observed in Fig. 4(a). Subsequently, 1180-nmcentered emission spanning approximately $1050-1300 \mathrm{~nm}$ was exhibited from $I=5-10 \mathrm{~mA}$ (Fig. 4(b)). Finally, the center wavelength blue-shifted up to $1100 \mathrm{~nm}$ and the emission span extended to $\sim 1000 \mathrm{~nm}$ for $I=150 \mathrm{~mA}$ (Fig. 4(c)). As summarized in Fig. 4(d), the peak wavelength (closed square) and emission band (solid bar) were blue-shifted, and the bandwidth was increased from approximately 70 to $120 \mathrm{~nm}$ with increased $I$. These behaviors 
can be attributed to state-filling effect, where supplied carriers fill the discrete states in the QD in a stepwise manner, from lower to higher energy states. Although the observed emission spectra cannot be certainly assigned to specific emission lines from each energy state of the four stacked QDs, the emission spanning $1200-1250 \mathrm{~nm}$ is attributed to electron/hole (e/h) recombination between GSs, and the emissions at approximately 1150$1200 \mathrm{~nm}$ and $1050-1150 \mathrm{~nm}$ is attributed to ES1 and ES2 recombinations, respectively. The number of states in the GS is lower than in the ES1 and ES2, and thus, GS emissions can be obtained, even though a low injection current $(\sim 3 \mathrm{~mA})$ is applied. Then, the injection current was increased, and the contribution of ES1 emissions began. When their intensity was almost equal to the GS intensity under the injection current of approximately $12.5 \mathrm{~mA}$, the bandwidth was increased to $120 \mathrm{~nm}$. On further increasing the injection current, the ES1 emissions were increased while the GS emissions were saturated; then, ES2 emissions were sequentially increased while the ES1 and GS emissions were saturated.

In order to clarify the state-filling effect, EL intensities at several wavelengths in the spectra are plotted as functions of $I$, as shown in Fig. 5. Ideally, the integral EL intensities of each QD energy state in each layer should be plotted; however, it is difficult to distinguish the emission spectra because of the broad and overlapping nature of emissions from QD ensembles, as described above. We thus utilized the plotted EL intensities as an approximate investigation. The EL intensities at 1200 and $1250 \mathrm{~nm}$, which should be dominantly contributed from the GS emissions, were increased for lower $I(<\sim 30 \mathrm{~mA})$ and then became gradually saturated for $I>\sim 30 \mathrm{~mA}$. On the other hand, the EL intensities at 1150 and 1100 $\mathrm{nm}$ increased sequentially after the increment of EL intensities at longer wavelength were decreased. These increments of EL intensities in order of wavelength (state energy) indicate the state-filling in the discrete states, which indicates QD-originated emission. Furthermore, the ratio of the saturation currents of the EL intensities can be explained by varying the 
number of carriers filling the QD states. The ratio of the number of states for GS:ES1:ES2 in a parabolic potential is 2:4:6 (including spin degeneracy) ${ }^{30}$, and thus, the ratio of the injection currents necessary for filling the QD states should be 2:6:12 for GS, GS+ES1, and GS+ES1+ES2. In addition, when the carrier density increases, the Auger process will occur, and the actual current to saturate these states will increase. The saturation currents of the EL intensities shown in Fig. 5 vary with respect to the increase in current for filling the states, e.g., approximately $20 \mathrm{~mA}$ for the longest wavelength in contrast to above $140 \mathrm{~mA}$ for the shortest wavelength.

The wide extended emission wavelength implies the contribution of emission from ES2 and not only ES1 and GS of the QDs. In our previous work ${ }^{21,22}$, the emission wavelength was extended to approximately $\sim 1100 \mathrm{~nm}$ from a chip with single contact under a current density of $400 \mathrm{~A} / \mathrm{cm}^{2}$. By introducing the segmented contacts, it was possible to increase the current density up to $3000 \mathrm{~A} / \mathrm{cm}^{2}$ in a single segment, which is approximately 8 times higher than that for the previous device, and emission from the higher energy state (ES2) emerged.

\section{B. Gain Spectra}

Figure 6 presents the deduced net modal gain spectra under various Is (applied to a 1-mm long segment of the waveguide) in accordance with equation (1). The positive or negative values of the net modal gain indicate that the QDs function as optical gain or absorption media at the given wavelength, respectively. As clearly seen in the spectra, positive gain regions occur from approximately $1240 \mathrm{~nm}$, where the GS emissions were mainly observed, and extended continuously to shorter wavelength with increasing $I$. The positive gain region under $I$ of $12.5 \mathrm{~mA}$ was $\sim 1183-1237 \mathrm{~nm}$ and was extended to a range of $\sim 1076-1233 \mathrm{~nm}$ (bandwidth of $\sim 160 \mathrm{~nm}$ ) under $I$ of $150 \mathrm{~mA}$. The extension of the positive gain region with the increase in $I$ can be also attributed to the sequential state-filling effect from GS to ES2, 
which is demonstrated by the dependence of the EL intensities. These results demonstrate that the fabricated chip operates as a gain media because of the QDs. The maximum gain value at $I$ of $150 \mathrm{~mA}$ was approximately $6 \mathrm{~cm}^{-1}$. The span of the gain region and the maximum gain value were almost the same under Is higher than $150 \mathrm{~mA}$. This result might be due to the influence of the heat caused by high Is. By introducing a thermoelectric cooler and a pulsed current source, the maximum gain value can be further increased.

Because the emission center wavelength of each QD layer was shifted continuously by the SRL, the broad gain spectra appeared without apparent dips in the positive gain regions of the spectra in contrast to the QD-SLD including QD layers with identical emission center wavelengths of GS and ES. This smooth gain feature is useful in obtaining a broad emission spectrum with homogeneous intensities. The gain values assigned at excited states (ES1 and ES2) were higher than that from GS, which is probably due to the higher state degeneracy in the ESs compared with the GS. The possibility of amplified spontaneous emission, that is, stimulated emissions from the states, is proportional to the DOS for the transition of carriers (Fermi's golden rule). The results above indicate the effectiveness of the segmented contacts for deriving higher ES emission and a broadband-gain.

\section{OCT imaging}

A fabricated QD-SLD chip was evaluated as an OCT light source. As described in the Introduction, the axial resolution of OCT image is governed by the central wavelength and the bandwidth of the EL spectrum. Figure 7 (a) presents normalized EL spectra of a QD-SLD under various Is (10-100 mA). In order to estimate the axial resolution when the chip was used in OCT, the IFT EL spectra were deduced, as shown in Fig. 7(b). The IFT EL spectrum provide more accurate estimation of the OCT axial resolution than that from the formula indicated in the Introduction: $0.44 \times \lambda_{0}{ }^{2} / \Delta \lambda$, because this formula is defined for a low- 
coherence light source with a Gaussian spectrum in the frequency unit. According to the Wiener-Khinchin theorem, a Fourier relationship exists between the autocorrelation function and the power spectrum of the light source ${ }^{31}$. The autocorrelation, which corresponds to the point-spread function, governs the axial resolution of the OCT image; the axial resolution can be estimated using the full-width at half-maximum (FWHM) of the IFT EL (power) spectra. As observed in the Fig. 7(b), the FWHM decreased with increased $I$. In addition to the increase in the bandwidth of the power spectrum, the blue-shift of the peak wavelength resulted in a decrease of the axial resolution to $3.8 \mu \mathrm{m}$ (in air) with an increase of $I$ to 100 $\mathrm{mA}$. This estimated axial resolution is less than half of that of typical commercial OCT: $\sim 10$ $\mu \mathrm{m}$. In addition, no apparent side lobes appeared beside the main peak, which indicates that the ghost images (noise) resulting from the side lobe should be minimized in an OCT image when the QD-SLD is used as a light source.

OCT imaging with the QD-SLD was performed for a test sample: a cover-glass plate with a refractive index of 1.5 and a thickness of approximately $150 \mu \mathrm{m}$. To produce a twodimensional OCT image of the sample, depth profiles were obtained by scanning the sample along the in-plane direction. Then, the depth profiles were displayed in two dimensions with a gray scale of the signal intensity. Figure 8(a) presents the obtained OCT image. Two white lines are clearly seen in the image, which represent reflections at the upper and lower surfaces of the sample. The distance between the lines is approximately $220 \mu \mathrm{m}$, which is consistent with the optical path length, that is, the thickness of the cover-glass plate multiplied by its refractive index. Figure $8(\mathrm{~b})$ presents a depth profile along the red dashed line in the image. Two peaks resulting from reflections from the surfaces of the cover-glass plate appear in the depth profile. The FWHM of each peak is approximately $4 \mu \mathrm{m}$, and no apparent side lobes appeared beside the peak. These features are consistent with those of the coherence function shown in Fig. 7(b). The axial resolution of the OCT image was thus determined to be 
approximately $4 \mu \mathrm{m}$, which is half of that of our previous result using a QD-SLD with a single contact ${ }^{22}$. This improvement of the axial resolution results from the modification of the prepared contacts; the segmented contacts enable an increase of $J$ and extension of the emission wavelength range.

Based on the above results, we conclude that the QD-SLD with segmented contacts is a promising light source for high-resolution OCT imaging. In particular, the axial resolution below $4 \mu \mathrm{m}$ in air is expected to distinguish single cells in an OCT image, which is difficult to observe using a conventional OCT system.

\section{SUMMARY}

A SLD chip based on self-assembled InAs QDs with segmented contacts was developed. EL spectra revealed the extension of the emission band to shorter wavelength with increasing I. This behavior is due to the contribution of higher excited states of the QDs to the emission, which were derived from the state-filling effect under higher Is. The measured optical gain of the QDs using the segmented contact method revealed a gain bandwidth beyond $160 \mathrm{~nm}$ under $I$ of $150 \mathrm{~mA}$. The gain bandwidth was also extended to shorter wavelength, corresponding to the contributions of the ESs of the QDs. These results indicate the effectiveness of the segmented contacts for deriving emissions of higher excited states of QDs and broadband gain. Furthermore, OCT imaging was performed using the QD-SLD, and the potential of the QD-SLD as a light source for high-resolution OCT was demonstrated.

\section{ACKNOWLEDGEMENTS}

N.O. would like to thank the members of Richard Hogg's group at the University of Sheffield for their support and fruitful discussions about the experimental results. This study was partly supported by Grants-in-Aid for Scientific Research (KAKENHI) Grant Number 
25286052 and the Canon foundation. N.O. would like to acknowledge financial support received from Wakayama University to visit the University of Sheffield. The fabrication of the SLD chip was supported by the NIMS Nanofabrication Platform in the "Nanotechnology Platform Project" sponsored by the Ministry of Education, Culture, Sports, Science and Technology, Japan (MEXT). 


\section{REFERENCES}

${ }^{1}$ Huang, E. A. Swanson, C. P. Lin, J. S. Schuman, W. G. Stinson, W. Chang, M. R. Hee, T. Flotte, K. Gregory, C. A. Puliafito, and J. G. Fujimoto, Science 254, 1178 (1991).

${ }^{2}$ M. E. Brezinski, Optical Coherence Tomography: Principles and Applications (Academic Press, New York, 2006).

${ }^{3}$ M. S. Patterson, B. C. Wilson, D. R. Wyman, Lasers in Medical Science 6, 379 (1991).

${ }^{4}$ J. Welzel, Skin Res. Technol. 7, 1 (2001).

${ }^{5}$ W. Drexler, U. Morgner, F. X. Kärtner, C. Pitris, S. A. Boppart, X. D. Li, E. P. Ippen, and J. G. Fujimoto, Opt. Lett. 24, 1221 (1999).

${ }^{6}$ B. Povazay, K. Bizheva, A. Unterhuber, B. Hermann, H. Sattmann, A. F. Fercher, W. Drexler, A. Apolonski, W. J. Wadsworth, J. C. Knight, P. St. J. Russell, M. Vetterlein, and E. Scherzer, Opt. Lett. 27, 1800 (2002).

${ }^{7}$ Z. M. Wang ed., Self-assembled quantum dots, lecture notes in nanoscale science and technology series vol. 1. (Springer, New York, 2008).

${ }^{8}$ Y. Arakawa and H. Sakaki, Appl. Phys. Lett. 40, 939 (1982).

${ }^{9}$ L. Goldstein, F. Glas, J. Y. Marzin, M. N. Charasse, G. Le Roux, Appl. Phys. Lett. 47, 1099 (1985).

${ }^{10}$ D. Leonardo, M. Krishnamurthy, C. M. Reaves, and P. Petroff, Appl. Phys. Lett. 63, 3203 (1993).

11 Z. Z. Sun, D. Ding, Q. Gong, W. Zhou, B. Xu, Z. G. Wang, Optical Quant. Electron. 31, 1235 (1999).

${ }^{12}$ Z. Y. Zhang, Z. G. Wang, B. Xu, P. Jin, Zh. Sun, and F. Q. Liu, IEEE Photonics Technol. Lett. 16, 27 (2004).

13 M. Rossetti, A. Markus, A. Fiore, L. Occhi, and C. Velez, IEEE Photon. Technol. Lett. 17, 540 (2005). 
${ }^{14}$ S. K. Ray, K. M. Groom, M. D. Beattie, H. Y. Liu, M. Hopkinson, and R. A. Hogg, IEEE Photonics Technol. Lett. 18, 58 (2006).

15 P. D. L. Greenwood, D. T. D. Childs, K. M. Groom, B. J. Stevens, M. Hopkinson, and R. A. Hogg, IEEE J. Sel. Top. Quantum Electron. 15, 757 (2009).

16 Z. Y. Zhang, R. A. Hogg, X. Q. Lv, and Z. G. Wang, Adv. Opt. Photonics 2, 201 (2010).

${ }^{17}$ X. Li, P. Jin, Q. An, Z. Wang, X. Lv, H. Wei, J. Wu, J. Wu, Z. Wang, Nanoscale Res. Lett. 6, 625 (2011).

${ }^{18}$ N. Yamamoto, K. Akahane, T. Kawanishi, H. Sotobayashi, Y. Yoshioka, and H. Takai, Jpn. J. Appl. Phys. 51, 02BG08 (2012).

19 S. Chen, M. Tang, Q. Jiang, J. Wu, V. G. Dorogan, M. Benamara, Y. I. Mazur, G. J. Salamo, P. Smowton, A. Seeds, and H. Liu, ACS Photonics 1, 638 (2014).

${ }^{20}$ N. Ozaki, T. Yasuda, S. Ohkouchi, E. Watanabe, N. Ikeda, Y. Sugimoto, and R. A. Hogg, Jpn. J. Appl. Phys. 53, 04EG10 (2014).

${ }^{21}$ T. Yasuda, N. Ozaki, H. Shibata, S. Ohkouchi, N. Ikeda, H. Ohsato, E. Watanabe, Y. Sugimoto, R. A. Hogg, IEICE Trans. Electron. E-99, (2016).

${ }^{22}$ H. Shibata, N. Ozaki, T. Yasuda, S. Ohkouchi, N. Ikeda, H. Ohsato, E. Watanabe, Y. Sugimoto, K. Furuki, K. Miyaji, and R. A. Hogg, Jpn. J. Appl. Phys. 54, 04DG07 (2015).

${ }^{23}$ P. Blood, G. M. Lewis, P. M. Smowton, H. Summers, J. Thomson, and J. Lutti, IEEE J. Sel. Top. Quantum Electron. 9, 1275 (2003).

${ }^{24}$ K. Nishi, H. Saito, S. Sugou, and J.-S. Lee, Appl. Phys. Lett. 74, 1111 (1999).

${ }^{25}$ N. Ozaki, K. Takeuchi, Y. Hino, Y. Nakatani, T. Yasuda, S. Ohkouchi, E. Watanabe, H. Ohsato, N. Ikeda, Y. Sugimoto, E. Clarke, and R. A. Hogg, Nanomat. Nanotechnol. 4, 26 (2014)

${ }^{26}$ W. Drexler and J. G. Fujimoto eds., Optical Coherence Tomography: Technology and Applications (Springer, New York, 2008). 
27 A. F. Fercher, C. K. Hitzenberger, G. Kamp, and S.Y. El-Zaiat, Opt. Commun. 117, 43 (1995).

${ }^{28}$ G. Häusler and M. W. Lindner, J. Biomed. Opt. 3, 21 (1998).

${ }^{29}$ C. Dorrer, N. Belabas, J.-P. Likforman, and M. Joffre, J. Opt. Soc. Am. B 17, 1795 (2000).

30 A. Wojs, P. Hawrylak, S. Fafard, and L. Jacak, Phys. Rev. B. 54, 5604 (1996).

31 L. Cohen, IEEE Signal Proc. Lett. 5, 292 (1998). 


\section{Figure Captions}

Fig. 1. (a) Profile of a fabricated sample. (b) Schematic image and photograph of the segmented contacts fabricated on the QD-SLD chip.

Fig. 2. Schematic images of device driven with different lengths.

Fig. 3. Schematic illustration of SD-OCT setup including the fabricated QD-SLD chip.

Fig. 4. (a)-(c) EL spectra obtained from the fabricated QD-SLD chip under various Is applied to segment 1. (d) Plotted peak wavelength (closed square) and span (solid bar) of the EL emissions.

Fig. 5. EL intensity plotted for each wavelength as a function of $I$.

Fig. 6. Net-modal-gain spectra of the QD-SLD under various Is.

Fig. 7. (a) Normalized EL spectrum from a QD-SLD under Is of 10-100 mA. (b) Coherence function of the EL spectra shown in (a).

Fig. 8. (a) OCT image obtained from a cover-glass plate with a refractive index and thickness of approximately 1.5 and $150 \mu \mathrm{m}$, respectively. Surfaces of the cover-glass are indicated by black arrows. (b) Depth profile along the red dashed line in the OCT image. The FWHM of the peaks indicated by black arrows is $\sim 4 \mu \mathrm{m}$. 


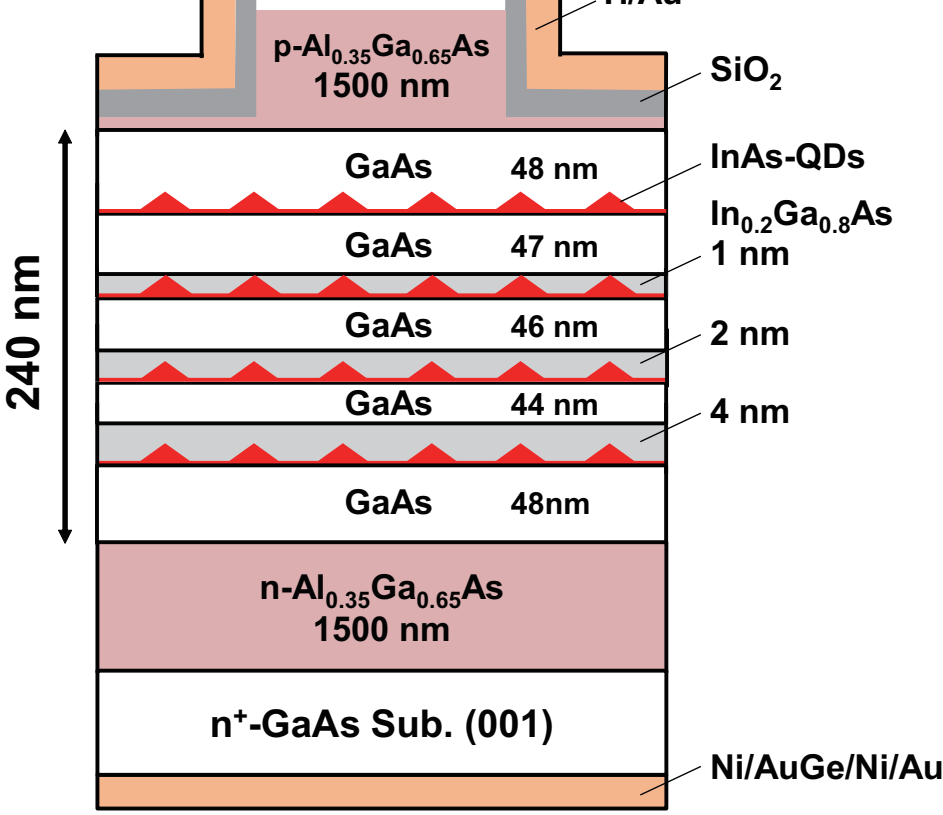

(b)

\section{Segmented contacts}

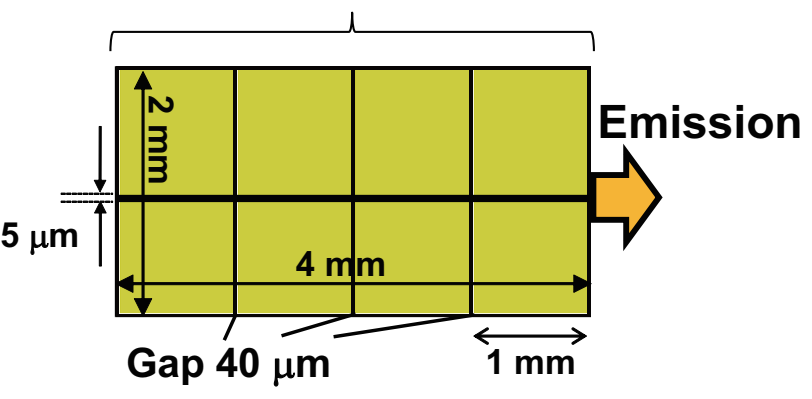




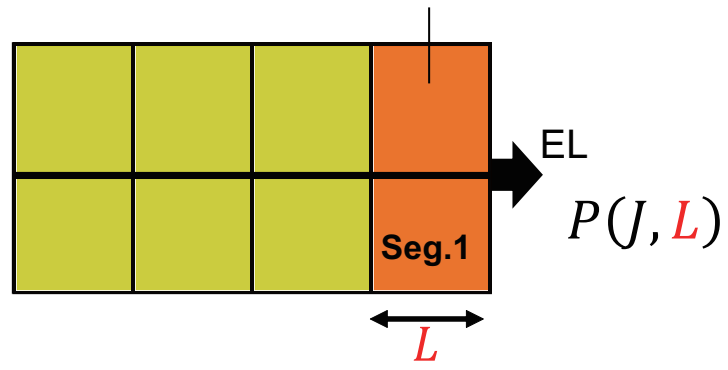

(b)

$\mathrm{J} \mathrm{A} / \mathrm{cm}^{2}$

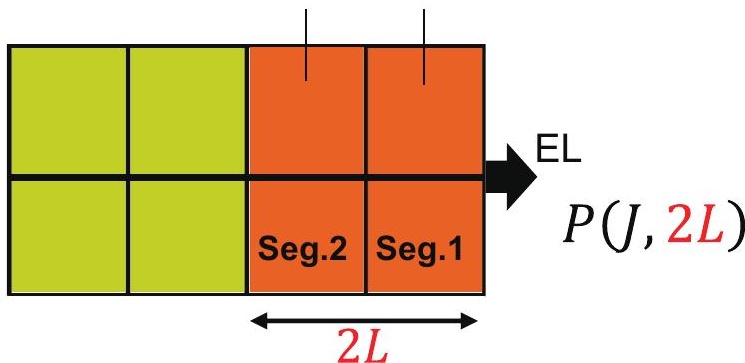



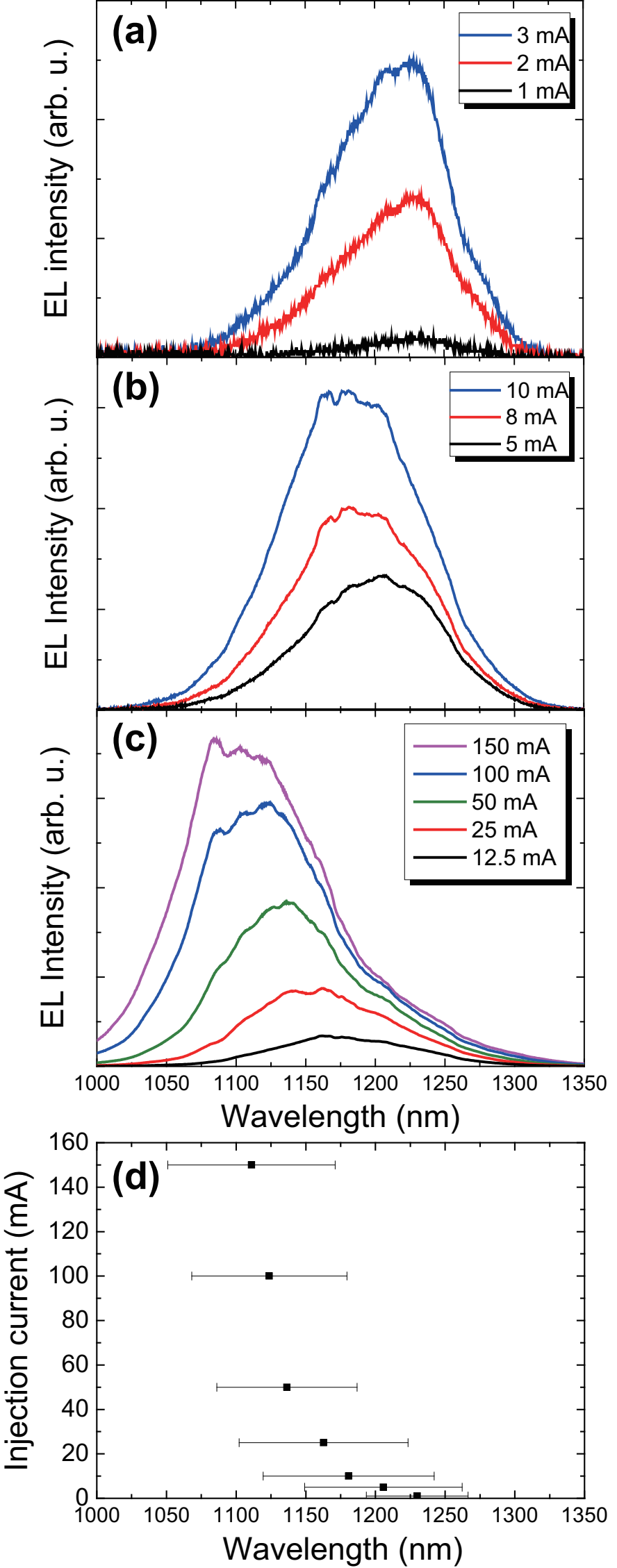


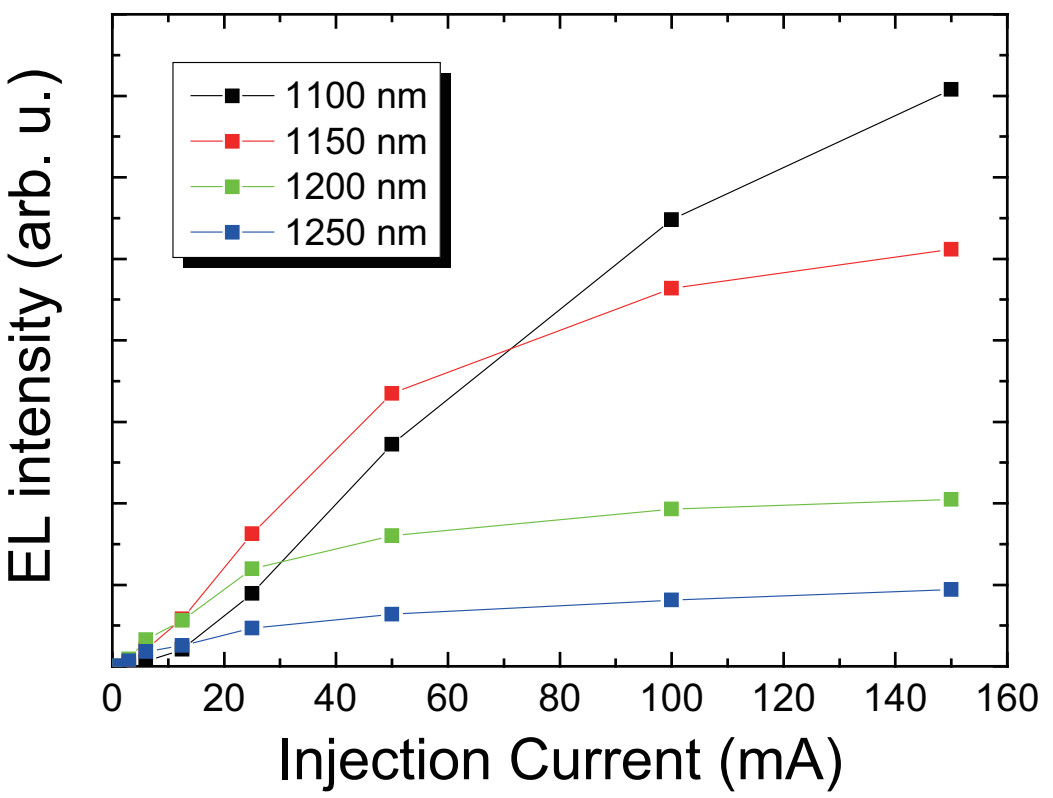




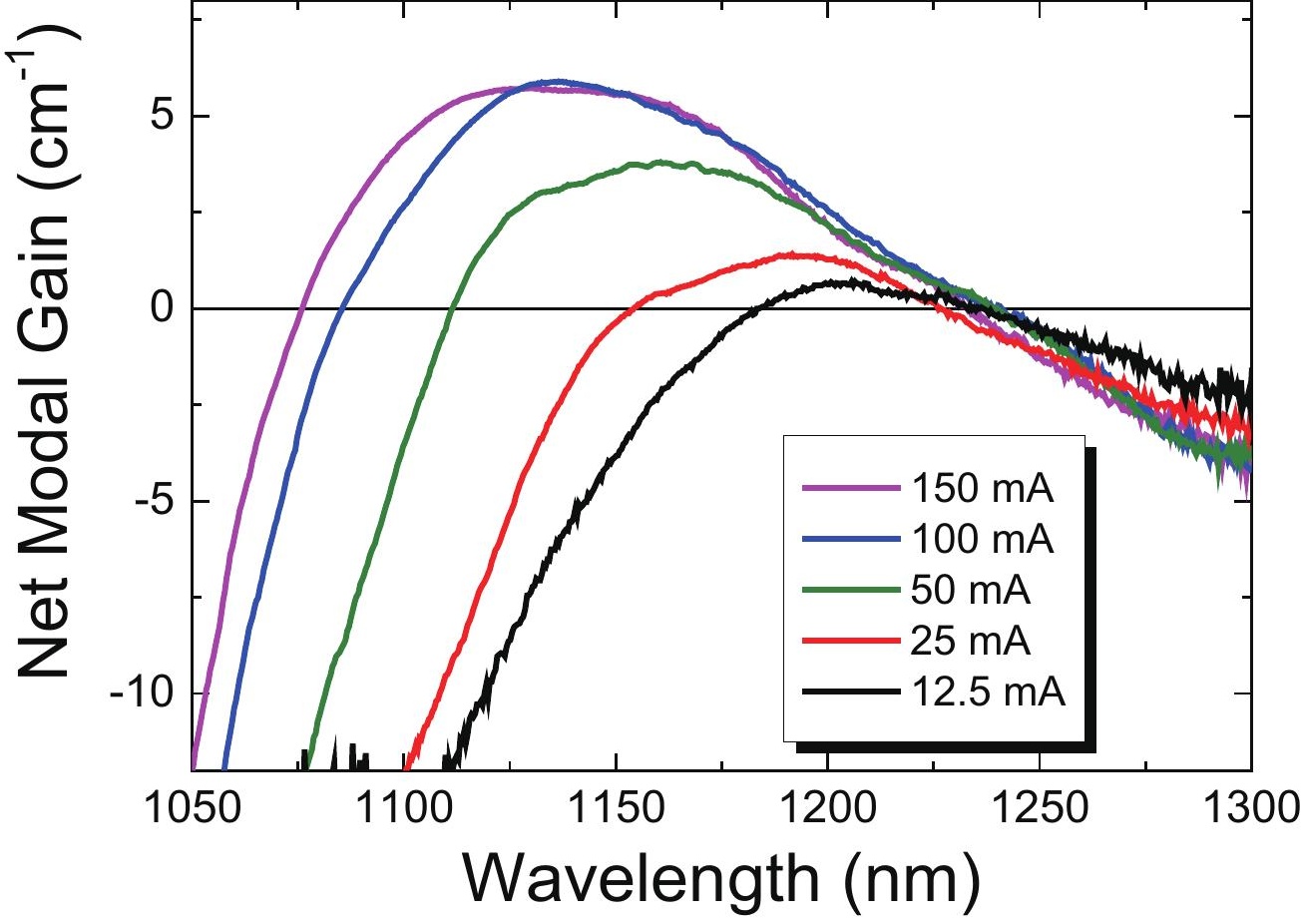



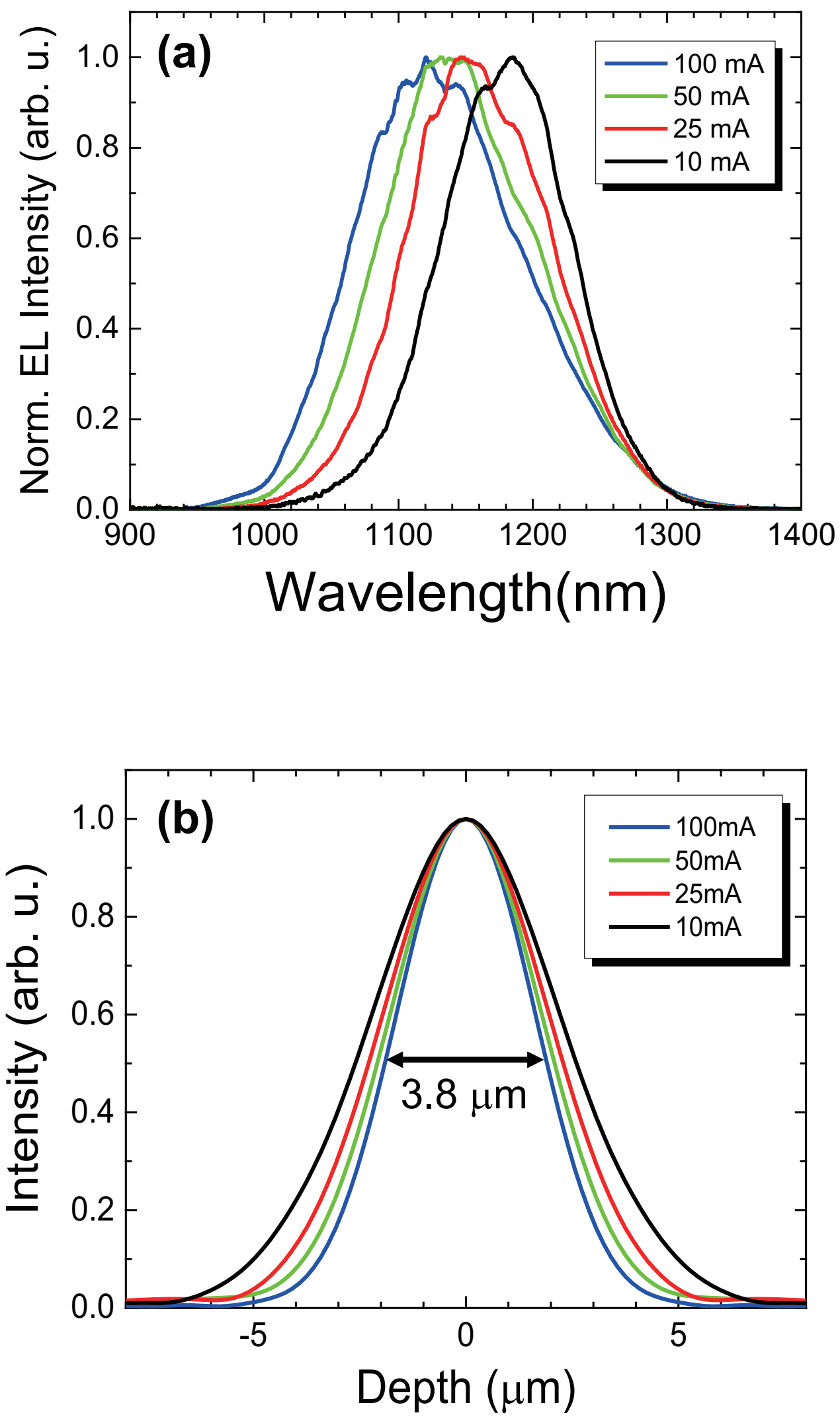


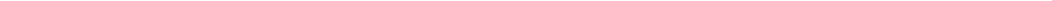

